\title{
MICROSCOPIC DEMONSTRATION OF COCCI IN THE CENTRAL NERVOUS SYSTEM IN EPIDEMIC POLIOMYELITIS
}

Ludig Hextone, George Mathers, and Leila Jackson From the Memorial Institute for Infections Diseases, Chicago

After reviewing recent observations on a coccus found in the central nervous system in epidemic poliomyelitis, ${ }^{1}$ one of us wrote as follows : ${ }^{2}$

"To conclude - the exact significance of this coccus in epidemic poliomyelitis cannot be determined now. The number of cases studied for its presence is too small to permit the conclusion that it occurs constantly in the disease or any form of the disease; in the few instances in which injections of culture have resulted in a condition indistinguishable from what is accepted as poliomyelitis in the monkey, the possibility that another and more important microbe may have been present cannot be excluded; the true poliomyelitic nature of the very interesting lesion caused by the coccus in rabbits has not been confirmed by proper tests on monkeys; and we lack also the results of extended immunization experiments. In any event a most interesting coccus has been found that merits study for its own sake as well as on account of the close relation its brief history bears to poliomyelitis."

In order to determine so far as possible whether coccal forms are demonstrable microscopically in the central nervous system in poliomyelitis as it occurs in different places, we obtained from various sources fixed poliomyelitic material, mostly pieces of spinal cord, which we have studied, and we now wish to make a brief report of the results.

On account of the variation in the time after death when the tissues had been fixed as well as on account of the different fixatives employed, uniform results have not been obtained with all the methods of staining that were used. For demonstration of cocci the Gram stain was the most satisfactory, but methylene blue and

Received for publication September 10, 1917.

1 Mathers: Jour. Am. Med. Assn., 1916, 67, p. 1019; Jour. Infec. Dis., 1917, 20, p. 113. Rosenow, Towne and Wheeter: Jour. Ant. Med. Assn., 1916, 67, p. 1202. Nuzum and Herzog: Ibid., p. 1205; Nuzum, ibid., p. 1437.

z Hektoen: Recent Investigations on the Bacteriology of Acute Poliomyelitis, Boston Med. and Surg. Jour., 1917, 176, p. 687. 


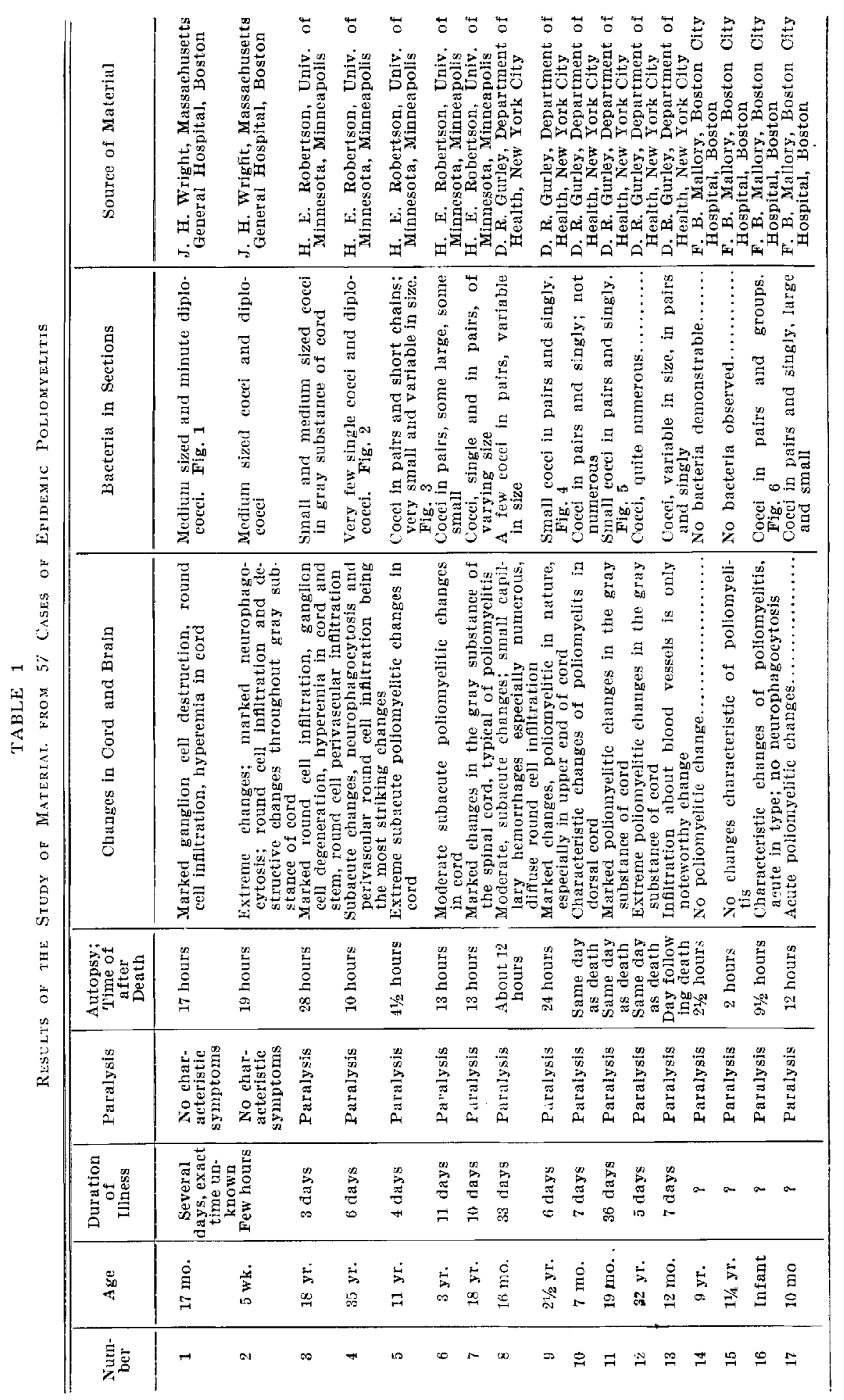




\begin{tabular}{|c|c|c|c|c|c|c|}
\hline 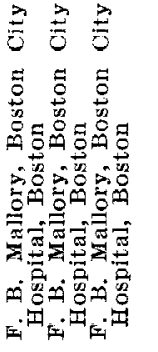 & 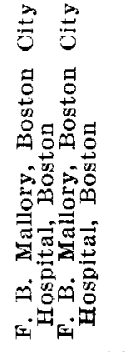 & 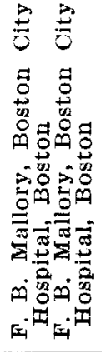 & 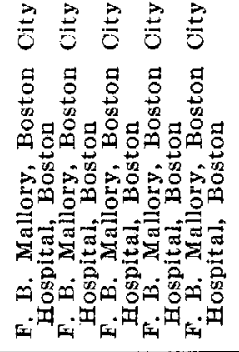 & 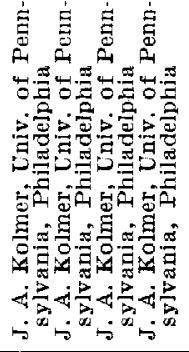 & 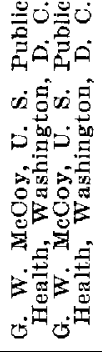 & 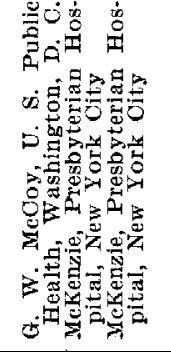 \\
\hline 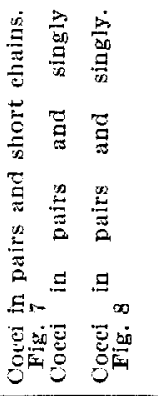 & 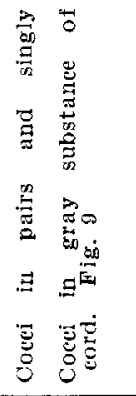 & 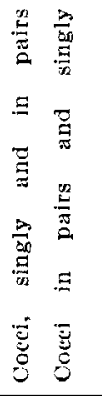 & 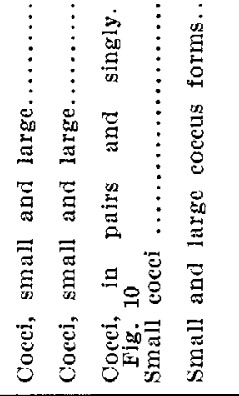 & 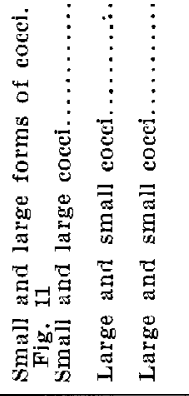 & 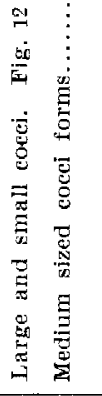 & 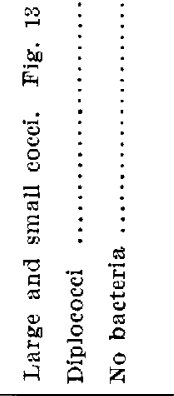 \\
\hline 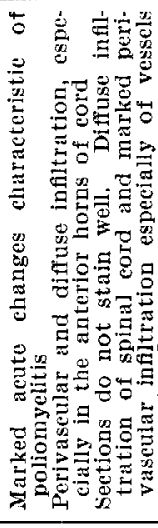 & 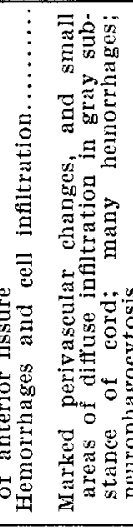 & 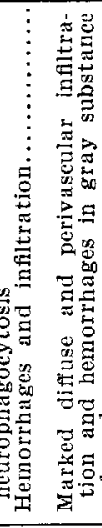 & 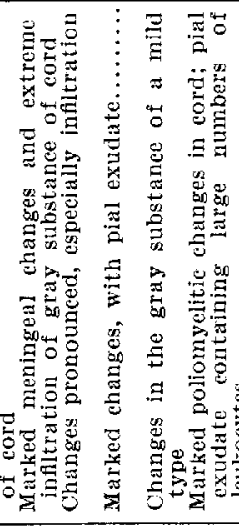 & 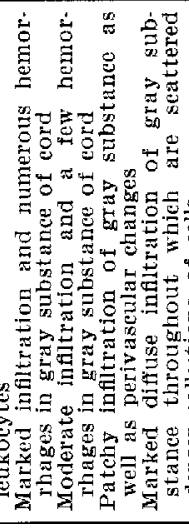 & 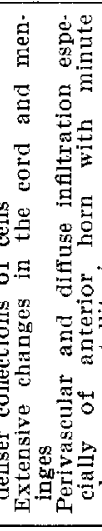 & 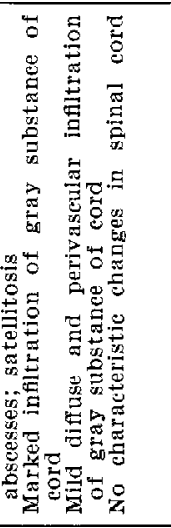 \\
\hline 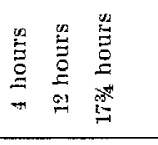 & 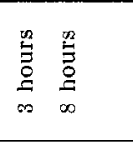 & 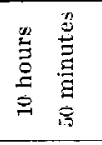 & 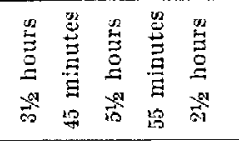 & 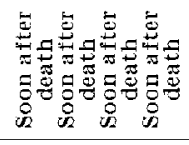 & 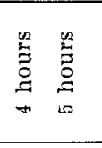 & 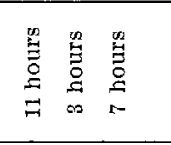 \\
\hline 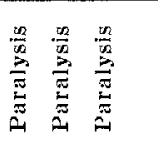 & 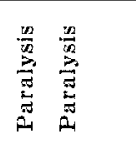 & 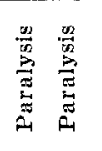 & 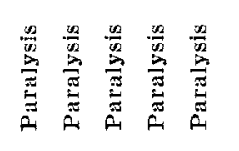 & 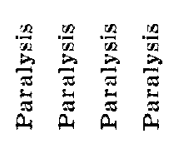 & 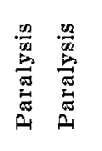 & 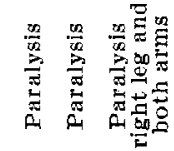 \\
\hline 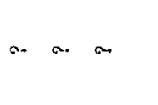 & $\infty \cdots$ & $\therefore \quad x$ & $\therefore$ & 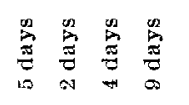 & 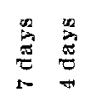 & 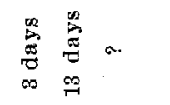 \\
\hline 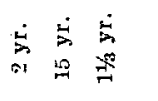 & 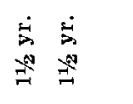 & $\begin{array}{l}\dot{5} \\
\dot{0} \\
0\end{array}$ & 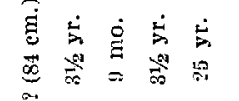 & 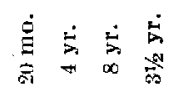 & 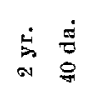 & 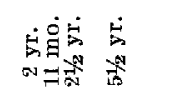 \\
\hline$m 8$ & 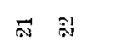 & $\pi$ & 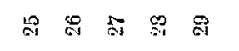 & 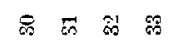 & 13 & m \\
\hline
\end{tabular}




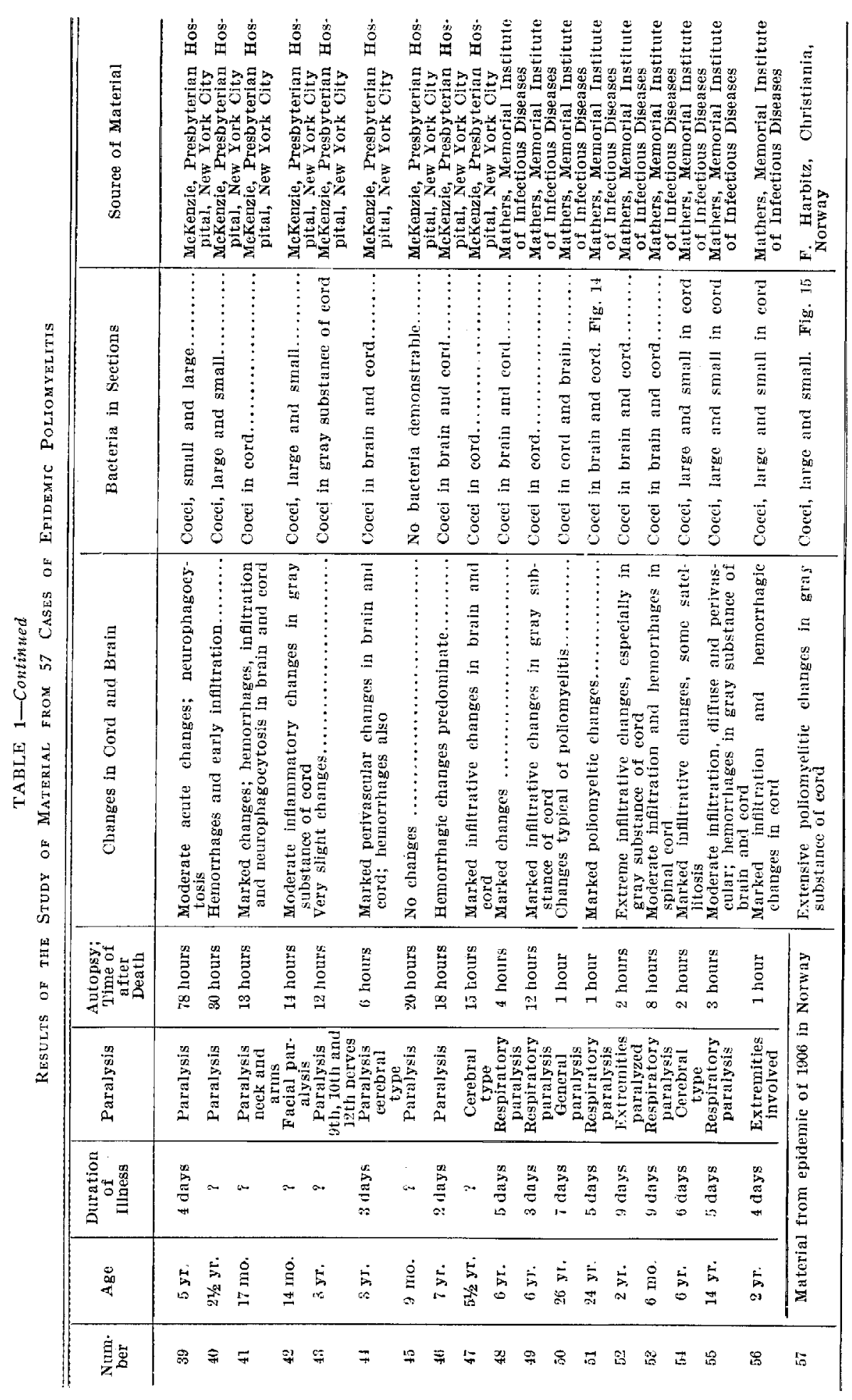


eosin and polychrome methylene blue and eosin also proved useful. The pieces were embedded in paraffin; the sections were from 5-10 microns thick.

In all, material from 57 cases has been studied (Table 1). Except in a few instances changes characteristic of epidemic poliomyelitis were present, most markedly in the gray matter, particularly the anterior horns, and in some cases, but to a much less extent, also in the membranes. The changes were hemorrhages, edema, and cellular infiltration, especially about the blood vessels; in most cases the perivascular infiltration was marked, and often associated with more diffuse infiltrations in the gray matter as well as dense focal accumulation of cells. Typical neurophagocytosis and extensive destruction of ganglion cells were present, but not in all the specimens; in a few cases the ganglion cells appeared to have been singled out for attack.

Definite coccal forms were found rather easily in sections showing typical poliomyelitic changes (Table 1 and Plates 1-3). They were single, oftener in pairs, occasionally in small clumps, usually outside but also within cells. They were located in the gray matter, in the walls of the blood vessels, in perivascular and other infiltrates, and in hemorrhagic areas; they were found also in meningeal infiltrations. These cocci correspond in general, so far as shape and size and staining by Gram's method are concerned, with the cocci recently isolated in cultures of the central nervous system in epidemic poliomyelitis. This statement is particularly applicable to Cases 48 to 56 , Table 1 , in which pure cultures of the coccus in mind were obtained by Mathers ${ }^{I}$ from the brain and cord. The cocci in the sections may vary in size, and larger and smaller forms may occur together. We did not find any other microbic forms than those described, and we found no such forms at all in tissues which did not show any changes.

\section{SUMMARY}

The cord and other parts of the central nervous system of about 50 instances of epidemic poliomyelitis, occurring in different parts of the country, have been found to contain in stained sections, cocci which look quite like the cocci that may be grown in cultures from the brain and cord in poliomyelitis. This result indicates that such cocci occur constantly in the central nervous system in epidemic poliomyelitis, and that their presence here is not explainable as due to accident or contamination. 
94 Ludvig Hektoen, Grorge Mathers, Leila Jackson

Plates 1, 2, 3

Magnification $1000 \mathrm{X}$. For details of the cases see Table 1.

Fig. 1.-Case 1.

Fig. 2.-Case 4.

Fig. 3.-Case 5 .

Fig. 4.-Case 9.

Fig. 5--Case 11.

Fig. 6.-Case 16.

Fig. 7.-Case 18.

Fig. 8.-Case 20.
Fig. 9.-Case 22.

Fig. 10.-Case 27.

Fig. 11,-Case 30.

Fig. 12.-Case 34.

Fig. 13.-Case 36.

Fig. 14.-Case 51.

Fig. 15.-Case 57. 
Plate 1

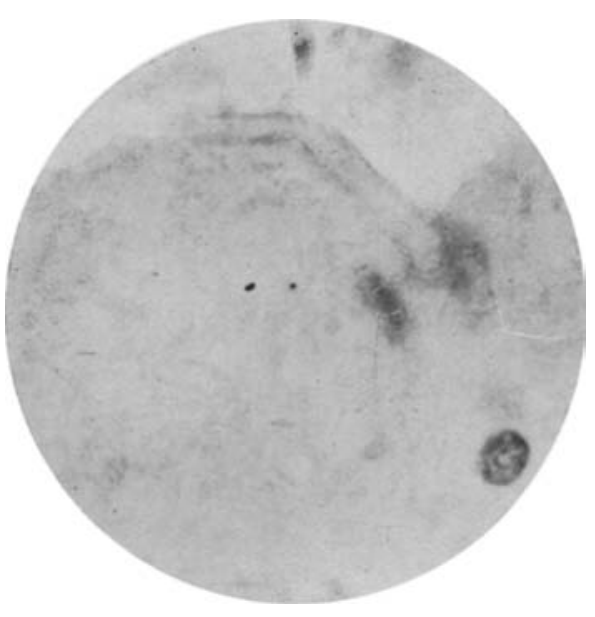

Fig. 1.

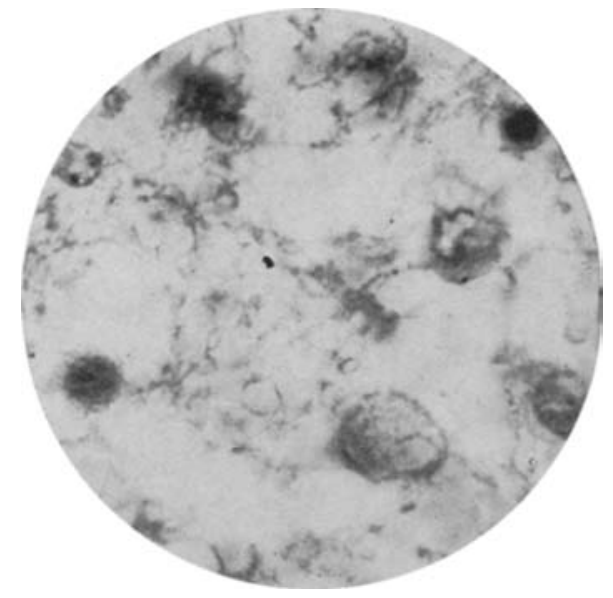

Fig. 2.

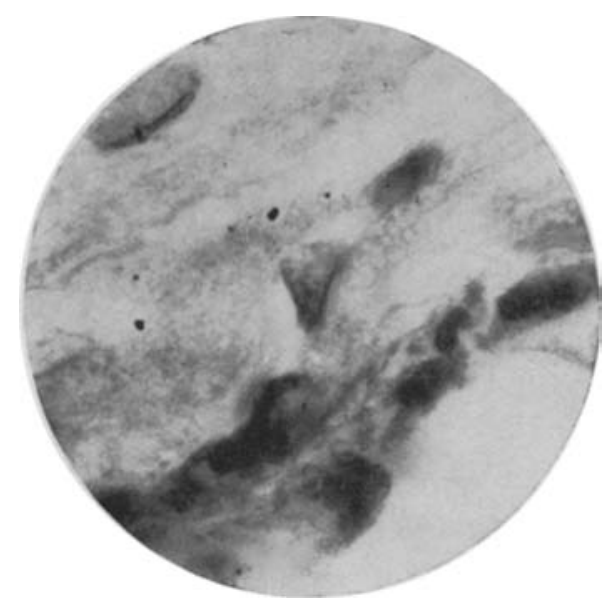

Fig. 3.

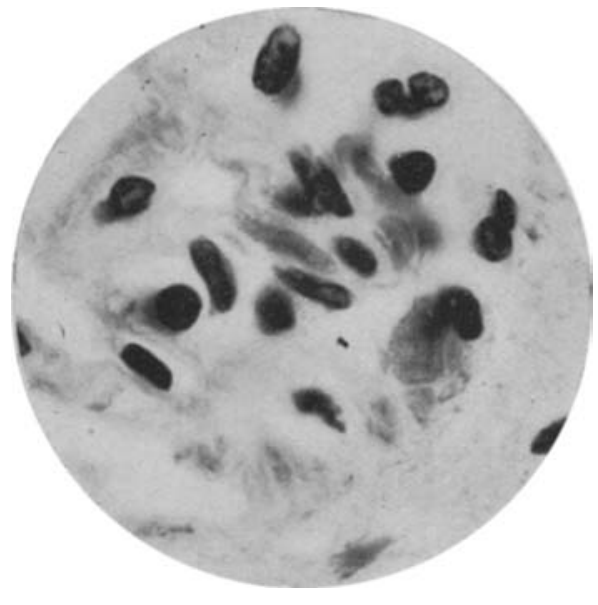

Fig. 4.

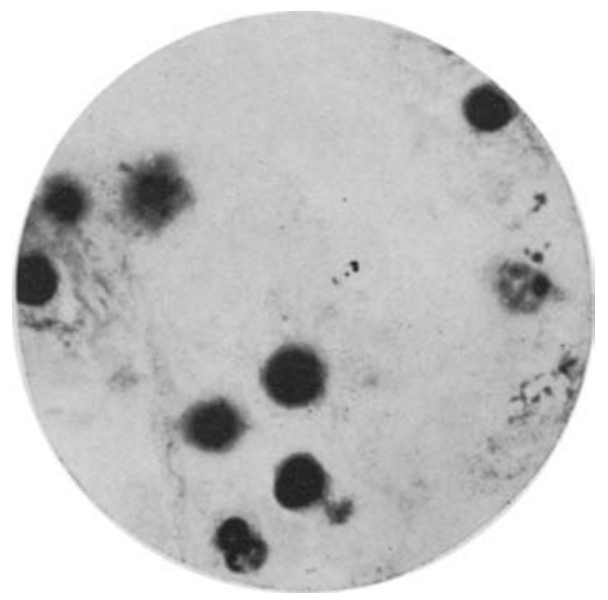

Fig. 5. 
Plate 2

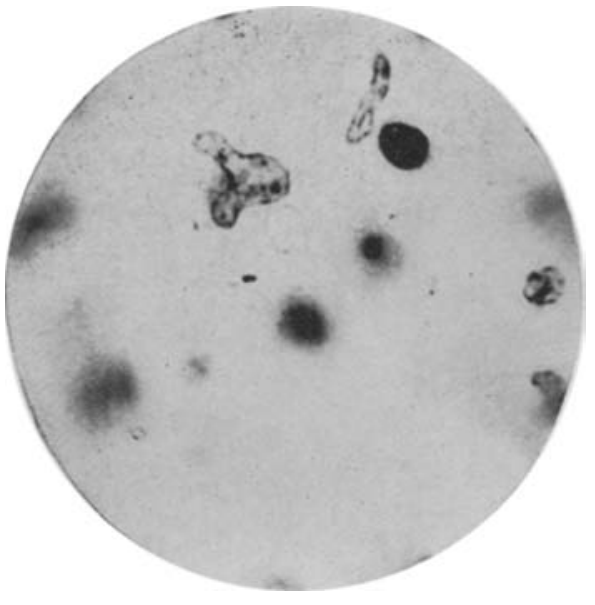

Iig. 6 .

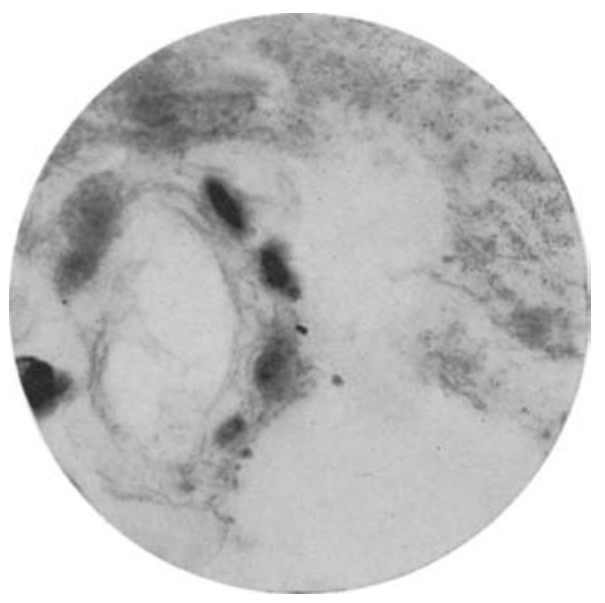

fig. 7.

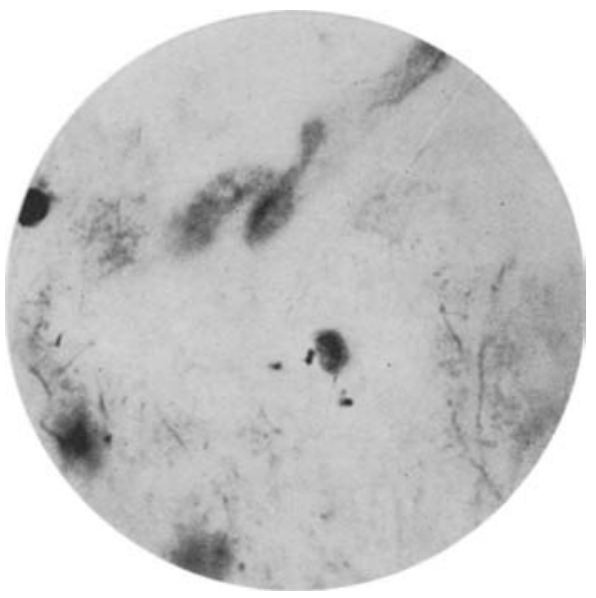

lïg. 8.

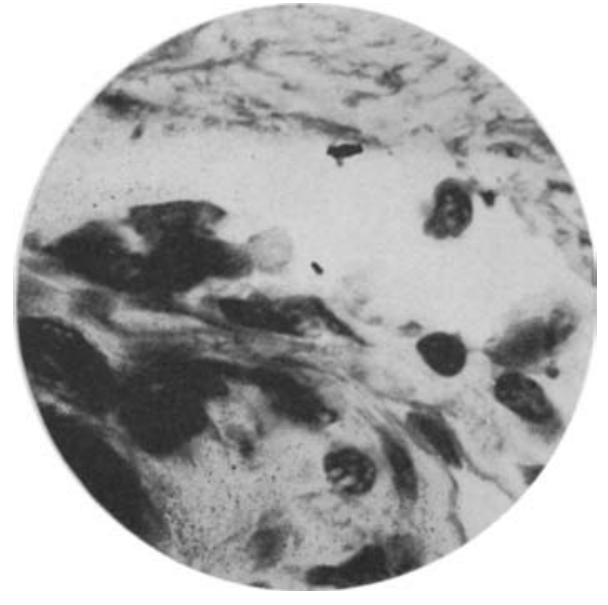

Fig. 9.

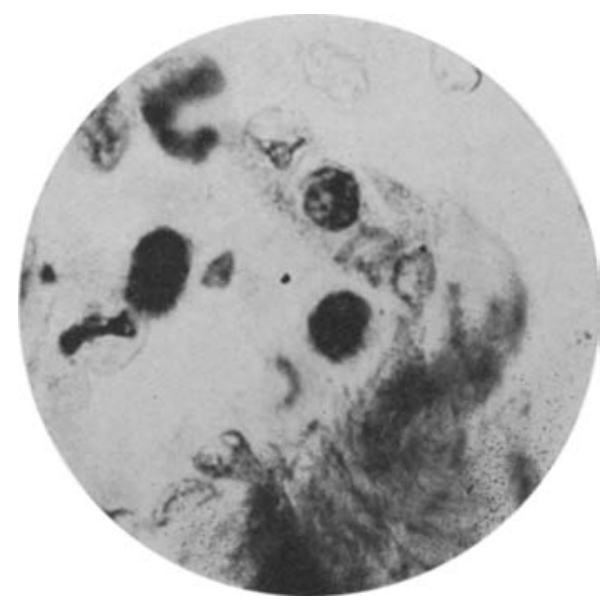

Fig, 10. 
Plate 3

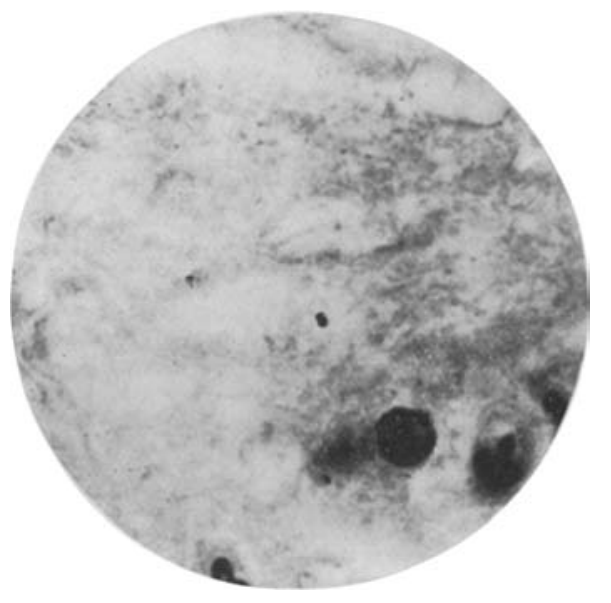

Fig. 11.

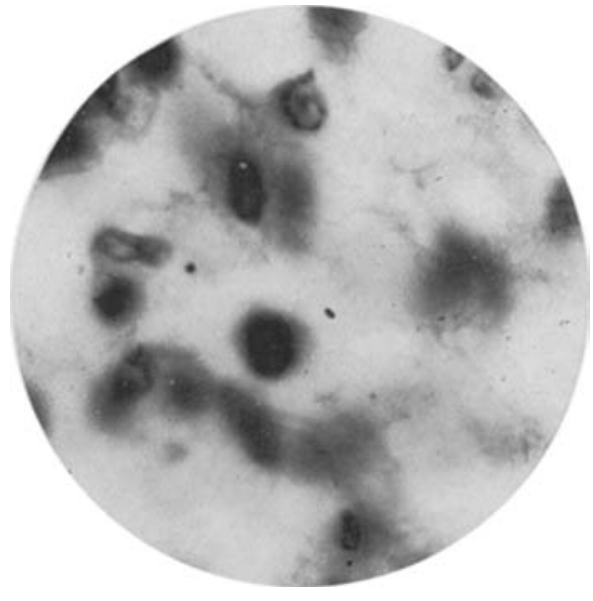

Fig. 12.

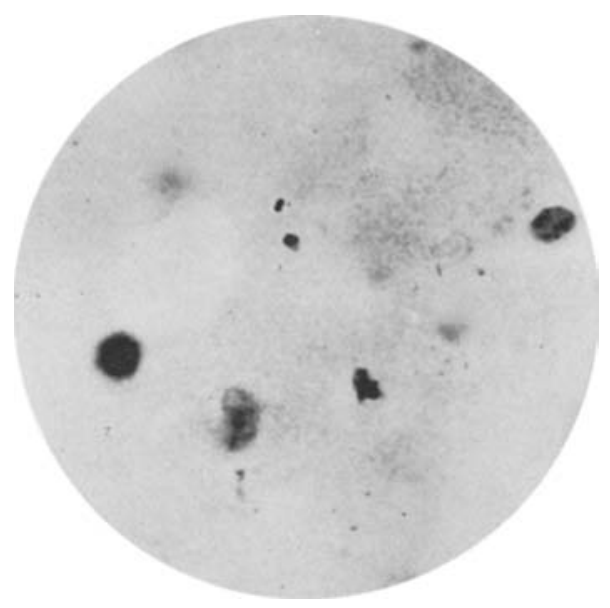

Fig. 13.

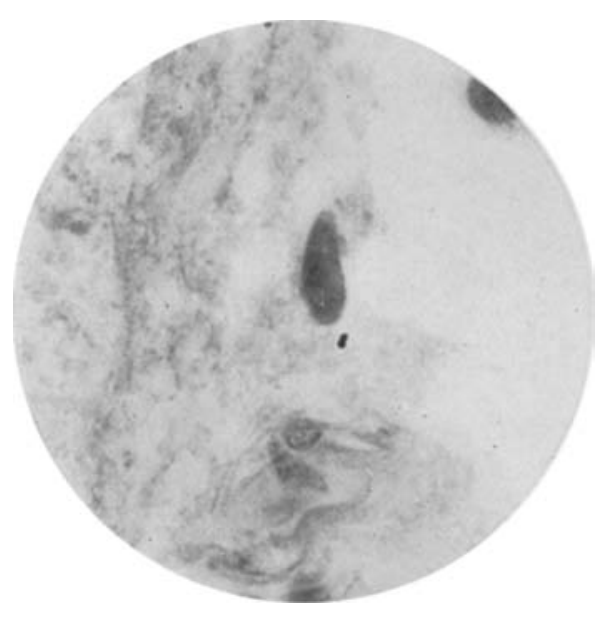

Fig. 14.

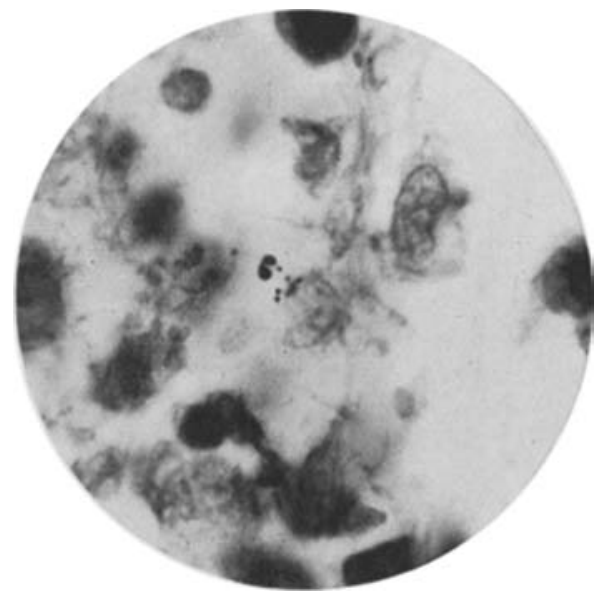

Fig. 15. 